\title{
Urinary Sodium Is a Potent Correlate of Proteinuria: Lessons from the Chronic Renal Insufficiency Cohort Study
}

\author{
Matthew R. Weir ${ }^{\mathrm{a}}$ Raymond R. Townsend ${ }^{\mathrm{b}}$ Jeffrey C. Fink ${ }^{\mathrm{a}}$ Valerie Teal $^{\mathrm{b}}$ \\ Stephen M. Sozio ${ }^{c}$ Cheryl A. Anderson ${ }^{c}$ Lawrence J. Appel ${ }^{c}$ Sharon Turban ${ }^{c}$ \\ Jing Chen ${ }^{d}$ Jiang He ${ }^{d}$ Natasha Litbarge ${ }^{e}$ Akinlolu Ojo ${ }^{f}$ Mahboob Rahmang \\ Leigh Rosen $^{b}$ Susan Steigerwalt ${ }^{\text {h }}$ Louise Strauss $^{g}$ Marshall M. Joffe ${ }^{b}$ \\ on behalf of the CRIC Study Investigators

\begin{abstract}
a University of Maryland School of Medicine, Baltimore, Md., bUniversity of Pennsylvania, Philadelphia, Pa.,
'Johns Hopkins Medical Institutions, Baltimore, Md., ' Tulane University School of Medicine, New Orleans, La., eUniversity of Illinois School of Medicine, Chicago, Ill., fUniversity of Michigan School of Medicine, Ann Arbor, Mich., ${ }^{9}$ Case Western Reserve School of Medicine, Cleveland, Ohio, and ${ }^{\mathrm{h}}$ St. Claire Specialty Center, Lakewood, Wash., USA
\end{abstract}

\section{Key Words \\ Proteinuria · Sodium • Potassium - Blood pressure}

\begin{abstract}
Background: While higher blood pressure is known to increase proteinuria, whether increased dietary sodium as estimated from 24-hour urinary excretion correlates with increased proteinuria in patients with chronic kidney disease (CKD) is not well studied. Methods: We measured 24-hour urinary sodium, potassium and protein excretion in 3,680 participants in the Chronic Renal Insufficiency Cohort study, to determine the relationship between urinary sodium and potassium and urinary protein excretion in patients with CKD. We stratified our data based on the presence or absence of diabetes given the absence of any data on this relationship and evidence that diabetics had greater urinary protein excretion at nearly every level of urinary sodium excretion. Multiple linear regressions were used with a stepwise inclusion of covariates such as systolic blood pressure, demographics, hemoglobin A1c and type of antihypertensive
\end{abstract}

medications to evaluate the relationship between urinary electrolyte excretion and proteinuria. Results: Our data demonstrated that urinary sodium (+1 SD above the mean), as a univariate variable, explained $12 \%$ of the variation in proteinuria $(\beta=0.29, p<0.0001)$, with rising urinary sodium excretion associated with increasing proteinuria. The significance of that relationship was only partially attenuated with adjustment for demographic and clinical factors and the addition of 24-hour urinary potassium to the model $(\beta=0.13$, $\left.\mathrm{R}^{2}=0.35, \mathrm{p}<0.0001\right)$. Conclusions: An understanding of the relationship between these clinical factors and dietary sodium may allow a more tailored approach for dietary salt restriction in patients with CKD.

Copyright $\odot 2012$ S. Karger AG, Basel

\section{Introduction}

Data from observational epidemiological studies and randomized controlled clinical trials demonstrate an association between dietary sodium intake and increased

\section{KARGER}

Fax +4161306 1234

E-Mail karger@karger.ch

www.karger.com
(C) 2012 S. Karger AG, Basel

0250-8095/12/0365-0397\$38.00/0

Accessible online at:

www.karger.com/ajn
Matthew R. Weir, MD

Division of Nephrology, University of Maryland School of Medicine Medical Center 22 S. Greene Street, Room N3W143

Baltimore, MD 21201 (USA)

E-Mail mweir@medicine.umaryland.edu 
blood pressure, with few exceptions [1-5]. Moreover, in small clinical studies in patients with diabetic and nondiabetic kidney disease and proteinuria, there is evidence that increasing dietary salt intake offsets both the antihypertensive and antiproteinuric effect of drugs that block the renin-angiotensin system $[6,7]$, whether used alone or in conjunction with thiazide diuretics [7]. The influence of other clinical and demographic factors which may affect the relationship between dietary salt and proteinuria has also not been well studied.

Higher dietary salt intake may affect the kidneys through both blood pressure- dependent and blood pressure-independent effects $[8,9]$. Although the mechanisms underlying the direct effect of dietary salt on the kidney are not well understood, experimental studies indicate that there may be a relationship between salt and endothelial dysfunction, primarily through an increase in oxidative stress [10], with less production of endogenous nitric oxide [11]. Structural changes of vascular beds in the brains of spontaneously hypertensive rats are also affected by dietary salt, independent of any change in systemic blood pressure [12].

Given the known relationships between increasing dietary salt and increasing blood pressure, particularly in individuals with chronic kidney disease (CKD), and the evidence from small clinical studies indicating that increasing dietary sodium intake offsets both the antihypertensive and antiproteinuric effects of drugs that block the renin-angiotensin system, it is important to understand the contribution of demographics, other medical comorbidities and antihypertensive treatment to the relationship between this important dietary electrolyte and proteinuria in a large cohort of patients with CKD. Thus, we performed multiple linear regressions in a large, wellcharacterized cohort to examine the possible influence of plausible demographic and clinical variables and antihypertensive treatment on the relationship between urinary sodium and 24-hour urinary protein excretion. These observations may be particularly important in formulating health care recommendations regarding diet in specific populations of individuals with CKD.

\section{Methods}

\section{Study Population}

The Chronic Renal Insufficiency Cohort (CRIC) study included a racially and ethnically diverse group of adults 21-74 years of age with both diabetic and nondiabetic kidney disease. Details on the assembly of the cohort are described elsewhere [13]. Inclusion in the CRIC study was based on age-specific estimated glomeru- lar filtration rate (eGFR) levels as follows: eGFR of $20-70 \mathrm{ml} /$ $\mathrm{min} / 1.73 \mathrm{~m}^{2}$ for participants aged $21-44$ years, $20-60 \mathrm{ml} / \mathrm{min} /$ $1.73 \mathrm{~m}^{2}$ for participants aged $45-64$ and $20-50 \mathrm{ml} / \mathrm{min} / 1.73 \mathrm{~m}^{2}$ for participants aged 65-74. A total of 3,939 participants were recruited into the CRIC study, 3,680 of whom had complete 24-hour urine collections. CRIC participants were recruited between June 2003 and March 2007 from 13 sites and 7 centers in the USA (Baltimore, Md.; Philadelphia, Pa.; Cleveland, Ohio; Ann Arbor/Detroit, Mich.; Chicago, Ill.; New Orleans, La., and Oakland/San Francisco, Calif.).

\section{Procedures}

At each CRIC study visit, several core measurements were ascertained and have been described elsewhere [13]. Most pertinent to this study, three brachial blood pressure measures were obtained in the sitting position after at least $5 \mathrm{~min}$ of quiet rest by trained and certified staff according to a standard protocol. A Tyco aneroid sphygmomanometer was used, with cuff size based on the participant's arm circumference. Participants were advised to refrain from coffee, tea or alcohol intake, cigarette smoking and vigorous exercise for at least $30 \mathrm{~min}$ before their examination. All blood pressure observers were certified for the blood pressure measurement protocol.

A 24-hour urine collection for protein and creatinine was obtained from each participant on the day of the blood pressure measures. Protocol-specified laboratory measurements were obtained from participants at each annual visit. Laboratory testing was performed at the central laboratory of the University of Pennsylvania. The eGFR was determined according to the abbreviated Modification of Diet in Renal Disease formula [14], using creatinine values calibrated to the Cleveland Clinic laboratory. Ethnicity was self-described by each participant.

\section{Statistical Analyses}

Continuous variables are presented as means \pm SD or $95 \%$ confidence intervals. Categorical variables are expressed as numbers (percentage). Certain variables (urinary sodium and clinical data) were prespecified in the basic model. In the analyses, we initially fit a joint model including both diabetics and nondiabetics. The log-transformed 24-hour urinary protein was designated as the outcome variable in this cross-sectional analysis, and measures including systolic blood pressure (SBP) and diastolic blood pressure, demographics, glycohemoglobin, type of antihypertensive medication(s) and urinary potassium were considered as key predictors, because of their possible relationship to proteinuria. Multiple linear regressions were performed sequentially. First, urinary sodium and clinical site were included in the model. Next, we included blood pressure, followed by several demographic and clinical variables known to be associated with proteinuria and designated as key confounders, including age, ethnicity, gender, eGFR, smoking status and waist circumference. Subsequently, glucose levels, glycohemoglobin, antihypertensive medications (diuretics, renin-angiotensin system blockers and calcium channel blockers), 24-hour urinary potassium and the urinary sodium to potassium ratio were entered into the regression model. We included the urinary sodium to potassium ratio as a variable, as prior studies have indicated that higher urinary potassium, especially in patients with higher urinary sodium, may be associated with lower blood pressure and target organ injury [15]. All analyses were subsequently stratified based on diabetic status, given the 
Table 1. Clinical and demographic characteristics

\begin{tabular}{|c|c|c|c|c|c|c|c|}
\hline & \multirow{2}{*}{$\begin{array}{l}\text { All } \\
(n=3,673)\end{array}$} & \multicolumn{3}{|l|}{ Nondiabetics } & \multicolumn{3}{|l|}{ Diabetics } \\
\hline & & $\begin{array}{l}\mathrm{UPr} \leq 0.15 \mathrm{~g} \\
(\mathrm{n}=1,094)\end{array}$ & $\begin{array}{l}\mathrm{UPr}>0.15 \mathrm{~g} \\
(\mathrm{n}=813)\end{array}$ & $\mathrm{p}$ & $\begin{array}{l}\mathrm{UPr} \leq 0.15 \mathrm{~g} \\
(\mathrm{n}=606)\end{array}$ & $\begin{array}{l}\mathrm{UPr}>0.15 \mathrm{~g} \\
(\mathrm{n}=1,160)\end{array}$ & $\mathrm{p}$ \\
\hline \multicolumn{8}{|l|}{ Demographics } \\
\hline Age, years & $58.4 \pm 10.9$ & $59.6 \pm 10.4$ & $54.1 \pm 12.8$ & $<0.001$ & $61.7 \pm 9.1$ & $58.5 \pm 9.8$ & $<0.001$ \\
\hline Male, $\mathrm{n}$ & $2,020(55)$ & $511(46.6)$ & $516(63.3)$ & $<0.001$ & $267(44)$ & $726(62.5)$ & $<0.001$ \\
\hline Non-Hispanic White & $1,590(43)$ & $615(56.1)$ & $345(42.3)$ & $<0.001$ & $269(44.3)$ & $361(31.1)$ & $<0.001$ \\
\hline Non-Hispanic Black & $1,530(42)$ & $387(35.3)$ & $358(43.9)$ & & $256(42.2)$ & $529(45.5)$ & \\
\hline Hispanic & $415(11)$ & $55(5)$ & $78(9.6)$ & & $60(9.9)$ & $222(19.1)$ & \\
\hline Other & $145(4)$ & $39(3.6)$ & $34(4.2)$ & & $22(3.6)$ & $50(4.3)$ & \\
\hline Current smoking, $\mathrm{n}$ & $479(13)$ & $125(11.4)$ & $146(17.9)$ & $<0.001$ & $51(8.4)$ & $157(13.5)$ & 0.002 \\
\hline Diuretics & $2,179(60)$ & $556(51)$ & $369(45.8)$ & 0.026 & $435(72)$ & $819(71)$ & 0.644 \\
\hline Calcium channel blockers & $1,488(41)$ & $321(29.4)$ & $344(42.7)$ & $<0.001$ & $233(38.6)$ & $590(51.1)$ & $<0.001$ \\
\hline \multicolumn{8}{|l|}{ Clinical and lab measures } \\
\hline Total cholesterol, mg/dl & $183.0 \pm 45.0$ & $188.2 \pm 39.2$ & $188.5 \pm 44.7$ & 0.858 & $170.7 \pm 40.5$ & $180.6 \pm 50.7$ & $<0.001$ \\
\hline Non-HDL cholesterol, mg/dl & $135.4 \pm 43.1$ & $136.3 \pm 37.6$ & $141.4 \pm 42.8$ & 0.007 & $124.3 \pm 39.2$ & $136.2 \pm 48.7$ & $<0.001$ \\
\hline $\mathrm{SBP}, \mathrm{mm} \mathrm{Hg}$ & $128.0 \pm 21.9$ & $120.3 \pm 18.5$ & $127.6 \pm 21.8$ & $<0.001$ & $123.0 \pm 18.5$ & $138.3 \pm 22.5$ & $<0.001$ \\
\hline DBP, mm Hg & $71.3 \pm 12.7$ & $70.5 \pm 11.4$ & $76.3 \pm 13.2$ & $<0.001$ & $65.0 \pm 11.1$ & $71.8 \pm 12.8$ & $<0.001$ \\
\hline $\mathrm{eGFR}, \mathrm{ml} / \mathrm{min} / 1.73 \mathrm{~m}^{2}$ & $42.8 \pm 13.4$ & $48.2 \pm 12.8$ & $40.1 \pm 13.6$ & $<0.001$ & $44.7 \pm 12.7$ & $38.8 \pm 12.3$ & $<0.001$ \\
\hline $\mathrm{eGFR}$ (CRIC), $\mathrm{ml} / \mathrm{min} / 1.73 \mathrm{~m}^{2}$ & $44.9 \pm 16.7$ & $53.4 \pm 17.3$ & $41.7 \pm 16.0$ & $<0.001$ & $46.5 \pm 15.9$ & $38.4 \pm 13.2$ & $<0.001$ \\
\hline Glucose, mg/dl & $114.9 \pm 51.2$ & $91.5 \pm 11.3$ & $91.6 \pm 11.9$ & 0.966 & $129.7 \pm 50.4$ & $145.5 \pm 69.3$ & $<0.001$ \\
\hline
\end{tabular}

Figures in parentheses represent percentages. UPr $=24$-Hour urinary protein; ARBs = angiotensin receptor blockers; $\mathrm{HDL}=$ highdensity lipoprotein; DBP = diastolic blood pressure.

sufficient numbers of participants to examine whether there may be differences in the relationship between urine electrolytes and proteinuria in diabetic versus nondiabetic kidney disease. Figure 1 was obtained from a locally weighted polynomial regression fit. Confidence intervals were computed locally assuming linear relationships, independent errors and normality. The value $\mathrm{R}^{2}$ is the proportion of variability that is accounted for by the model. All analyses were executed in SAS 9.2 (SAS Institute, Cary, N.C., USA).

\section{Results}

\section{Demographic Characteristics}

The entire CRIC cohort of 3,939 participants included 3,673 who had complete 24-hour urine collections (nondiabetic, $\mathrm{n}=1,907$; diabetic, $\mathrm{n}=1,766$ ). Table 1 shows the important demographic and clinical characteristics of the CRIC participants. Note that in the analytic cohort, the ethnicity percentage was similar between minority populations and non-Hispanic whites, and there were slightly more men. A large proportion of patients were on renin-angiotensin system-blocking drugs (69\%), and only $13 \%$ were current smokers.

The mean blood pressure of 128/71 $\mathrm{mm} \mathrm{Hg}$ indicated good control, but the mean waist circumference and body mass index were high (106 cm and 32, respectively). Mean eGFR was $42.8 \mathrm{ml} / \mathrm{min} / 1.73 \mathrm{~m}^{2}$, and mean 24-hour urinary protein was $790 \mathrm{mg}$.

Patients with proteinuria of more than $150 \mathrm{mg} / \mathrm{day}$ were more likely to be current smokers and receiving renin-angiotensin blockers and calcium channel blockers. In addition, they had higher non-high-density lipoprotein cholesterol and higher SBP and diastolic blood pressure and, if diabetic, higher glycosylated hemoglobin. They also had a lower eGFR.

Table 2 depicts the mean and median urinary sodium, urinary potassium and urinary sodium to potassium ra- 
Table 2. Urine laboratory data

\begin{tabular}{|c|c|c|c|c|c|c|c|}
\hline & $\begin{array}{l}\text { All } \\
(\mathrm{n}=3,673)\end{array}$ & \multicolumn{3}{|l|}{ Nondiabetics } & \multicolumn{3}{|l|}{ Diabetics } \\
\hline Mean $\pm \mathrm{SD}$ & $162.0 \pm 77.6$ & $146.2 \pm 73.3$ & $171.2 \pm 80.1$ & $<0.001$ & $158.8 \pm 71.6$ & $172.0 \pm 80.4$ & $<0.001$ \\
\hline Median & 150.9 & 135.3 & 161.1 & $<0.001$ & 148.6 & 162.0 & 0.002 \\
\hline IQR & $108.5-201.6$ & $94.5-182.5$ & $117.3-214.2$ & & $109.7-198.8$ & $115.7-213.4$ & \\
\hline Median & 51.3 & 52.5 & 50.1 & 0.312 & 49.6 & 51.9 & 0.061 \\
\hline IQR & $37.5-68.6$ & $35.6-69.2$ & $36.7-67.1$ & & $38.3-67.9$ & $39.0-69.1$ & \\
\hline \multicolumn{8}{|c|}{ Urinary sodium/potassium ratio } \\
\hline Mean $\pm \mathrm{SD}$ & $3.3 \pm 1.6$ & $3.0 \pm 1.7$ & $3.6 \pm 1.7$ & $<0.001$ & $3.2 \pm 1.4$ & $3.3 \pm 1.5$ & 0.198 \\
\hline Median & 3.0 & 2.7 & 3.2 & $<0.001$ & 3.0 & 3.1 & 0.344 \\
\hline IQR & $2.2-4.0$ & $1.9-3.8$ & $2.3-4.4$ & & $2.3-3.9$ & $2.3-4.0$ & \\
\hline
\end{tabular}

$\mathrm{UPr}=24$-Hour urinary protein; $\mathrm{IQR}=$ interquartile range.

tio in the CRIC participants. This analysis was considered as there is some evidence that increased dietary potassium may offset some of the adverse effects of dietary sodium [15]. The table also divides the participants into nondiabetics and diabetics and splits them further into two groups based on whether their 24-hour urinary protein excretion is less than or equal to $150 \mathrm{mg}$ per day or above this level. Both nondiabetics and diabetics had a similar prevalence of increased urinary sodium excretion in the groups with more than $150 \mathrm{mg}$ of protein per day. Urinary potassium excretion did not differ in the nondiabetics across the levels of proteinuria. However, in diabetics, the more proteinuric participants excreted slightly more potassium in their urine compared to the group with less proteinuria $(p=0.06)$. Likewise, the sodium to potassium ratio in the urine was statistically greater in nondiabetics with more than $150 \mathrm{mg} /$ day proteinuria $(p<0.001)$, whereas in diabetics, there was no difference in the sodium to potassium ratio based on the amount of proteinuria.

Figure 1 depicts the unadjusted data of urinary protein excretion in grams per $24 \mathrm{~h}$ compared to urinary sodium excretion (millimoles per $24 \mathrm{~h}$ ) for both diabetics and nondiabetics. Note the similar upward trend between the unadjusted urinary sodium excretion and 24-hour urinary protein excretion in both diabetics and nondiabetics. Overall, diabetics had greater 24 -hour urinary protein excretion at almost every level of urinary sodium excretion except at the highest levels (greater than 350 $\mathrm{mmol} / 24 \mathrm{~h}$ ).

\section{Regression Model}

Table 3 shows our basic model including urinary sodium and clinical site. These two covariates in the joint model for participants with a urinary sodium excretion value more than $1 \mathrm{SD}$ above the norm yielded similar $\beta$ coefficients and $\mathrm{R}^{2}$ values for both diabetics and nondiabetics, indicating a consistent positive association between urinary sodium and proteinuria across the populations studied. Yet, as a univariate variable, urinary sodium explained only $12 \%$ of the variation in proteinuria. Progressive adjustment for demographic and clinical variables did not remove the cross-sectional relationship between urinary sodium and proteinuria. This relationship persisted for both diabetics and nondiabetics. We describe the influence of demographic and clinical variables in the next few paragraphs.

In the joint model, with the addition of SBP to the model, one can see a slight decrease in the $\beta$ coefficient relating urinary sodium to proteinuria. Similar changes occurred in both diabetic and nondiabetic populations. There was a substantial increase in $\mathrm{R}^{2}$ with the inclusion of SBP for both diabetic and nondiabetic populations, indicating that SBP explains some of the variability.

With the subsequent stepwise inclusion of demographic variables for the whole cohort, a decline in the $\beta$ coefficient to 0.15 was observed. These changes were similar in both nondiabetics ( 0.28 to 0.18$)$ and diabetics $(0.24$ to 0.11 ). Within the demographic category, female gender was associated with a negative slope, whereas non-Hispanic blacks and Hispanics had a significant positive slope. It is possible that these slope differences may asso- 
Fig. 1. Unadjusted data of 24-hour urinary protein excretion in relation to 24 -hour urinary excretion of sodium for both diabetics and nondiabetics. Confidence intervals are represented by thin lines. Vertical lines at the bottom of the figure indicate the numbers of patients.

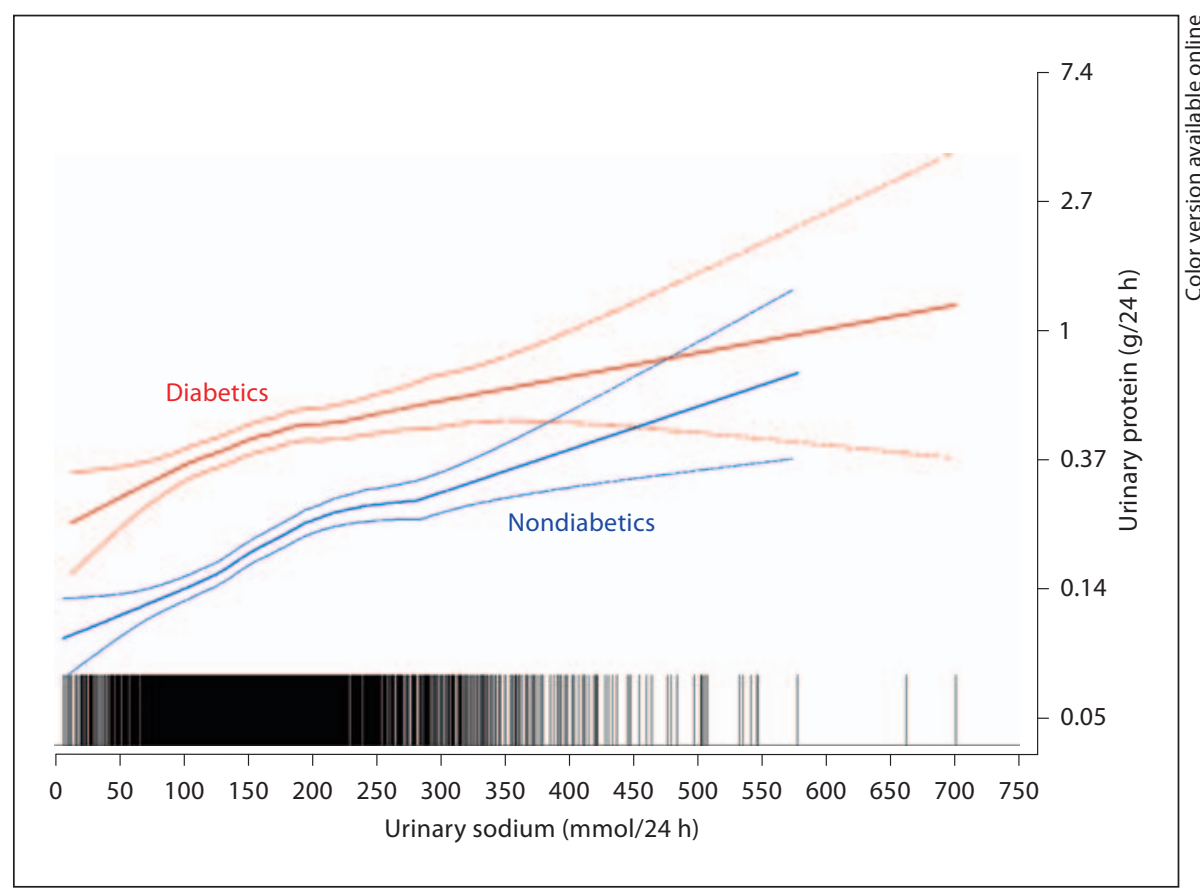

Table 3. Multivariable regression model to examine the relationship between 24-hour urinary sodium and log-transformed 24-hour urinary protein

\begin{tabular}{|c|c|c|c|c|c|c|c|c|c|}
\hline \multirow[t]{2}{*}{ Model } & \multicolumn{3}{|c|}{ All $(n=3,673)$} & \multicolumn{3}{|c|}{ Nondiabetics $(\mathrm{n}=1,904)$} & \multicolumn{3}{|c|}{ Diabetics $(n=1,766)$} \\
\hline & estimate & $\mathrm{p}$ & $\mathrm{R}^{2}$ & estimate & $\mathrm{p}$ & $\mathrm{R}^{2}$ & estimate & $\mathrm{p}$ & $\mathrm{R}^{2}$ \\
\hline Urinary sodium/SD, CRIC site only & 0.287 & $<0.0001$ & $11.6 \%$ & 0.292 & $<0.0001$ & $12.2 \%$ & 0.234 & $<0.0001$ & $8.7 \%$ \\
\hline \multicolumn{10}{|l|}{ Addition of } \\
\hline SBP & 0.272 & $<0.0001$ & $21.1 \%$ & 0.282 & $<0.0001$ & $15.0 \%$ & 0.238 & $<0.0001$ & $22.9 \%$ \\
\hline Demographics, SBP & 0.146 & $<0.0001$ & $31.4 \%$ & 0.181 & $<0.0001$ & $25.1 \%$ & 0.113 & 0.0014 & $35.5 \%$ \\
\hline Demographics, SBP, glucose & 0.151 & $<0.0001$ & $34.6 \%$ & 0.192 & $<0.0001$ & $25.3 \%$ & 0.119 & 0.0009 & $36.5 \%$ \\
\hline Demographics, SBP, glucose, urinary potassium & 0.132 & $<0.0001$ & $34.6 \%$ & 0.192 & $<0.0001$ & $25.3 \%$ & 0.075 & 0.0648 & $36.7 \%$ \\
\hline Demographics, SBP, glucose, medications & 0.145 & $<0.0001$ & $35.1 \%$ & 0.181 & $<0.0001$ & $26.6 \%$ & 0.115 & 0.0013 & $37.2 \%$ \\
\hline $\begin{array}{l}\text { No urinary sodium, demographics, SBP, } \\
\text { glucose, medications }\end{array}$ & -0.434 & $<0.0001$ & $34.3 \%$ & -0.369 & $<0.0001$ & $25.1 \%$ & -0.513 & $<0.0001$ & $36.8 \%$ \\
\hline
\end{tabular}

ciate with differential risk for progression of renal disease.

With the subsequent inclusion of glucose measures in the joint model overall, the $\beta$ coefficient increases slightly. Similar modest increases in the $\beta$ coefficient with glucose measures occur in both nondiabetics and diabetics. In this section of the joint model, glycosylated hemoglobin was a more important predictive value for the $\beta$ coefficient than the single glucose measurement. Thus, glucose measures did not contribute substantially to explain the relationship between urinary sodium and proteinuria.

Urinary Sodium and Potassium

Correlates of Proteinuria
Next, we included antihypertensive medications in the model. In the joint model, one can see little change in the $\beta$ coefficient relating urinary sodium to proteinuria. This was true for both nondiabetics and diabetics. There was also a difference in the $\beta$ coefficient for the whole cohort, as well as the diabetics, when we compared calcium channel blockers to diuretics and angiotensin-converting enzyme (ACE) inhibitors, suggesting differential glomerular hemodynamic effects of these medications. There were similar changes in the joint model for both diabetics and nondiabetics. 
In the next model, we evaluated the influence of 24hour urinary potassium excretion. This addition had little impact on the association between urinary sodium and proteinuria. The $\beta$ coefficient and $\mathrm{R}^{2}$ values are negligibly changed, indicating little influence of urinary potassium on the relationship of urinary sodium to proteinuria. We saw minimal influence of the sodium to potassium ratio as well (data not shown).

At the bottom of table 3, we removed urinary sodium from the model to examine the independent relationship of each demographic and clinical variable with proteinuria. Of note, one can see the powerful relationship of SBP and demographic factors with proteinuria independent of urinary sodium.

\section{Discussion}

Secondary analyses of clinical trials and observational studies in patients with CKD have demonstrated the importance of proteinuria as a predictor of adverse renal outcomes [16-18]. Moreover, epidemiological studies have demonstrated the value of proteinuria as a biomarker of increased risk for cardiovascular events [19]. Clinical studies have demonstrated that increasing dietary sodium intake is associated with increasing blood pressure and increasing proteinuria in patients with CKD $[6,7$, $20-22]$. The goal of our study was to evaluate the crosssectional relationship between 24-hour measurements of urinary sodium and potassium and proteinuria in almost 3,700 participants in the CRIC study. We stratified our analysis based on the presence or absence of diabetes as there were no prior studies examining this relationship, and there could be potential differences of clinical relevance. In addition, as illustrated in figure 1, diabetic patients exhibited greater 24-hour urinary protein excretion at almost every level of urinary sodium. In this analysis, we demonstrate that there is a correlation between urinary sodium excretion and 24-hour urinary protein. However, our results indicate that urinary sodium (+1 SD above the mean) as a single variable only explains $12 \%$ of the variation in proteinuria. Moreover, with the stepwise inclusion of other demographic variables, the $\beta$ coefficient declines, indicating that the apparently independent contribution of 24-hour urinary sodium is reduced after considering a variety of demographic and clinical factors. Similarly, with the removal of urinary sodium from the model, one can see the powerful influence of SBP and demographic factors on 24 -hour urinary protein measures.
Previous small studies have demonstrated that increasing urinary sodium excretion, as a measure of dietary sodium ingestion, is associated with an attenuation of the antihypertensive and antiproteinuric effects of drugs that block the renin-angiotensin system, such as ACE inhibitors or angiotensin receptor blockers $[6,7]$. This is evident whether these medicines are used alone or in conjunction with thiazide diuretics [7]. However, none of these studies adjusted for clinical and demographic factors as we have, nor did they have such a large number of patients with both diabetic and nondiabetic kidney disease. Our cross-sectional study does not provide specific information on whether or not a high level of urinary sodium excretion may attenuate the effects of reninangiotensin system blockade on proteinuria. What it does provide are a number of interesting associations between urinary sodium and protein excretion in patients with CKD, taking into account several clinical and demographic variables. When adjusting for clinical and demographic factors, there was a reduced contribution of urinary sodium to 24 -hour urinary protein excretion. We suspect that this may in part be related to the well-controlled blood pressure in our participants (mean 128/71 $\mathrm{mm} \mathrm{Hg}$ ). If greater dietary salt intake increases proteinuria through blood pressure-dependent effects, conceivably it would be quenched under circumstances of more effective control of blood pressure. However, one could also view that the persistence of the relationship between urinary sodium excretion and proteinuria despite adjustment for confounders suggests that there are blood pressure-independent effects of salt on glomerular permeability to proteins or renal tubular epithelial cell uptake mechanisms. Some investigators have suggested that increasing dietary salt may induce endothelial dysfunction through oxidative stress and diminish the production of endogenous nitrous oxide [23]. Likewise, other investigators have demonstrated the effect of increasing dietary salt on vascular beds in the brain in hypertensive rats independent of changes in blood pressure [9]. Thus, there may be vascular effects of dietary salt which occur despite well-treated blood pressure.

Amongst the demographic variables, it is notable that female gender was associated with a lesser risk of proteinuria, whereas non-Hispanic Blacks and Hispanics had significantly more proteinuria. These factors were important in diminishing the association of urinary sodium excretion and urinary protein excretion. Not surprisingly, SBP, which was associated with more proteinuria, diminished the contribution of urinary sodium excretion to 24 -hour urinary protein when added to the model. He- 
moglobin A1c was correlated with increased proteinuria but added little to the overall model with stepwise inclusion. There were also differences in the association of urinary sodium excretion with proteinuria based on the different antihypertensive medications. Both diabetics and nondiabetics had greater $\beta$ coefficients with calcium channel blockers than they did with diuretics or ACE inhibitors. This may be related to the effects of calcium channel blockers in preferentially dilating the afferent glomerular arteriole. Overall, however, the use of antihypertensive medications did not alter the association of urinary sodium excretion and proteinuria. Similarly, measurement of 24-hour urinary potassium excretion did not appear to alter the contribution of urinary sodium to urinary protein excretion. It has been suggested that increasing dietary potassium ingestion can offset the antihypertensive effects of increased dietary sodium [15]. It is possible that with higher levels of blood pressure than those observed in our participants $(128 / 71 \mathrm{~mm} \mathrm{Hg})$, one could see a potential vasodepressor response to increased potassium ingestion. It is also possible that with higher blood pressures, increasing dietary potassium could be associated with reduced urinary protein excretion. Our patients received no specific dietary counseling during this cohort study except that offered during the course of their routine clinical care.

Our results have some limitations. It is important to consider that the CRIC population that we studied is not necessarily representative of the US CKD population. We describe only a cross-sectional relationship between urinary sodium and urinary protein excretion after adjustment for a variety of clinical and demographic factors. Urinary electrolyte excretion may not be an accurate representation of dietary electrolyte consumption. In addition, only a single 24 -hour urine collection was available. Others have described that 3 overnight urine collections may be a more accurate way to calculate dietary ingestion of sodium [24]. However, the advantage of our study is the large number of cross-sectional measures in both diabet- ics and nondiabetics with well-controlled blood pressure, many of whom were receiving renin-angiotensin systemblocking therapy. Thus, these observations may provide perspective for the relative association of dietary salt and proteinuria in patients with CKD whose blood pressure is well controlled on a renin-angiotensin-blocking regimen. As others have suggested $[25,26]$, dietary sodium modification may be an important consideration in many, if not most, patients with CKD.

Our results suggest that in the aggregate, there is a relationship between 24-hour urinary sodium excretion and proteinuria. We clearly demonstrate that a number of clinical and demographic factors reduce the contribution of dietary sodium to 24 -hour protein excretion. A more complete understanding of the longitudinal relationship between dietary electrolytes as reflected in urinary electrolyte excretion is needed. This may allow a more rational approach to diet modification as a means of facilitating proteinuria reduction in patients with CKD.

\section{Acknowledgements}

This work was supported by the following institutions: University of Maryland GCRC (M01 RR-16500), University of Pennsylvania CTRC CTSA (UL1 RR-024134), The Johns Hopkins University (UL1 RR-025005), Case Western Reserve University Clinical and Translational Science Collaborative (University Hospitals of Cleveland, Cleveland Clinical Foundation and MetroHealth UL1 RR-024989), University of Michigan (GCRC grant M01 RR000042, CTSA grant UL1 RR-024986), University of Illinois at Chicago CTSA (UL1RR029879), Tulane/LSU/Charity Hospital General Clinical Research Center (RR-05096) and Kaiser NIH/ NCRR UCSF-CTSI (UL1 RR-024131).

We thank Tia A. Paul, University of Maryland School of Medicine, Baltimore, Md., for expert secretarial support.

\section{Disclosure Statement}

No conflicts of interest to declare.
References

Urinary Sodium and Potassium

Correlates of Proteinuria
1 Bibbins-Domingo K, Chertow GM, Coxson PG, Moran A, Lightwood JM, Pletcher MJ, et al: Projected effect of dietary salt reductions on future cardiovascular disease. N Engl J Med 2010;362:590-599.

-2 Graudal NA, Galloe AM, Garred P: Effects of sodium restriction on blood pressure, renin, aldosterone, catecholamines, cholesterols, and triglyceride: a meta-analysis. JAMA 1998;279:1383-1391.
3 Midgley JP, Matthew AG, Greenwood CM, Logan AG: Effect of reduced dietary sodium on blood pressure: a meta-analysis of randomized controlled trials. JAMA 1996;275: 1590-1597.

-4 Smith-Spangler CM, Juusola JL, Enns EA, Owens DK, Garber AM: Population strategies to decrease sodium intake and the burden of cardiovascular disease: a cost-effectiveness analysis. Ann Intern Med 2010;152:481-483. 
5 Stolarz-Skrzypek K, Kuznetsova T, Thijs L, Tikhonoff V, Seidlerova J, Richart T, et al: Fatal and nonfatal outcomes, incidence of hypertension, and blood pressure changes in relation to urinary sodium excretion. JAMA 2011;305:1777-1785.

6 Heeg JE, de Jong PE, van der Hem GK, de Zeeuw D: Efficacy and variability of the antiproteinuric effect of ACE inhibition by lisinopril. Kidney Int 1989;36:272-279.

7 Vogt L, Waanders F, Boomsma F, de ZD, Navis G: Effects of dietary sodium and hydrochlorothiazide on the antiproteinuric efficacy of losartan. J Am Soc Nephrol 2008; 19 : 999-1007.

8 Messerli FH, Schmieder RE, Weir MR: Salt. A perpetrator of hypertensive target organ disease? Arch Intern Med 1997;157:24492452.

9 Tobian L, Hanlon S: High sodium chloride diets injure arteries and raise mortality without changing blood pressure. Hypertension 1990;15:900-903.

$\checkmark 10$ Weir MR, Dengel DR, Behrens MT, Goldberg AP: Salt-induced increases in systolic blood pressure affect renal hemodynamics and proteinuria. Hypertension 1995;25: 1339-1344.

-11 Jones-Burton C, Mishra SI, Fink JC, Brown J, Gossa W, Bakris GL, et al: An in-depth review of the evidence linking dietary salt intake and progression of chronic kidney disease. Am J Nephrol 2006;26:268-275.

12 Perry IJ, Beevers DG: Salt intake and stroke: a possible direct effect. J Hum Hypertens 1992;6:23-25.
13 Feldman HI, Appel LJ, Chertow GM, Cifelli D, Cizman B, Daugirdas J, et al: The Chronic Renal Insufficiency Cohort (CRIC) Study: design and methods. J Am Soc Nephrol 2003; 14(7 suppl 2):S148-S153.

14 Peterson JC, Adler S, Burkart JM, Greene T, Hebert LA, Hunsicker LG, et al: Blood pressure control, proteinuria, and the progression of renal disease. The Modification of Diet in Renal Disease Study. Ann Intern Med 1995; 123:754-762.

15 D’Elia L, Barba G, Cappuccio FP, Strazzullo P: Potassium intake, stroke, and cardiovascular disease: a meta-analysis of prospective studies. J Am Coll Cardiol 2011;57:12101219.

16 Astor BC, Matsushita K, Gansevoort RT, van d, V, Woodward M, Levey AS, et al: Lower estimated glomerular filtration rate and higher albuminuria are associated with mortality and end-stage renal disease. A collaborative meta-analysis of kidney disease population cohorts. Kidney Int 2011;79:13311340 .

17 de Zeeuw D, Remuzzi G, Parving HH, Keane WF, Zhang Z, Shahinfar S, et al: Proteinuria, a target for renoprotection in patients with type 2 diabetic nephropathy: lessons from RENAAL. Kidney Int 2004;65:2309-2320.

18 Jafar TH, Stark PC, Schmid CH, Landa M, Maschio G, Marcantoni C, et al: Proteinuria as a modifiable risk factor for the progression of non-diabetic renal disease. Kidney Int 2001;60:1131-1140.
9 de Zeeuw D, Remuzzi G, Parving HH, Keane WF, Zhang Z, Shahinfar S, et al: Albuminuria, a therapeutic target for cardiovascular protection in type 2 diabetic patients with nephropathy. Circulation 2004;110:921-927.

20 Myrvang H: Progression of renal disease: high salt intake blunts the benefit of ACE inhibitors and accelerates renal function decline. Nat Rev Nephrol 2012;8:61.

21 Vegter S, Perna A, Postma MJ, Navis G, Remuzzi G, Ruggenenti P: Sodium intake, ACE inhibition, and progression to ESRD. J Am Soc Nephrol 2012;23:165-173.

$22 \mathrm{Yu}$ W, Luying S, Haiyan W, Xiaomei L: Importance and benefits of dietary sodium restriction in the management of chronic kidney disease patients: experience from a single Chinese center. Int Urol Nephrol 2012;44: 549-556.

23 Nakamura T, Kataoka K, Tokutomi Y, Nako H, Toyama K, Dong YF, et al: Novel mechanism of salt-induced glomerular injury: critical role of eNOS and angiotensin II. J Hypertens 2011;29:1528-1535.

24 Liu K, Dyer AR, Cooper RS, Stamler R, Stamler J: Can overnight urine replace 24hour urine collection to asses salt intake? Hypertension 1979;1:529-536.

25 Krikken JA, Laverman GD, Navis G: Benefits of dietary sodium restriction in the management of chronic kidney disease. Curr Opin Nephrol Hypertens 2009;18:531-538.

26 Wright JA, Cavanaugh KL: Dietary sodium in chronic kidney disease: a comprehensive approach. Semin Dial 2010;23:415-421. 